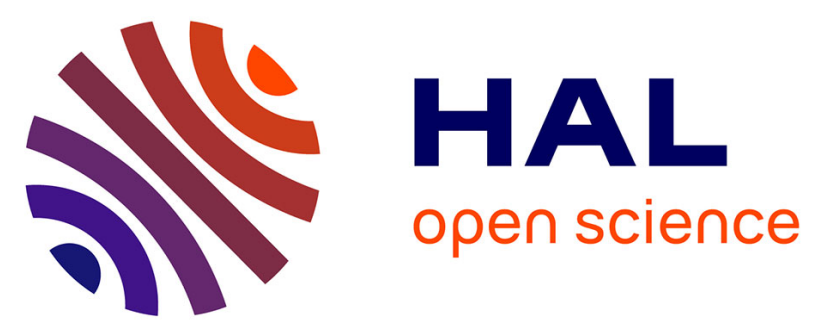

\title{
IShTAR: a test facility to study the interaction between RF wave and edge plasmas
}

\author{
I. Shesterikov, K. Crombé, A. Kostic, D. A. Sitnikov, Mariia Usoltceva, R. \\ Ochoukov, Stéphane Heuraux, Jérôme Moritz, Eric Faudot, F. Fischer, et al.
}

\section{- To cite this version:}

I. Shesterikov, K. Crombé, A. Kostic, D. A. Sitnikov, Mariia Usoltceva, et al.. IShTAR: a test facility to study the interaction between RF wave and edge plasmas. Review of Scientific Instruments, 2019, 90, pp.083506. 10.1063/1.5092431 . hal-02264258

\section{HAL Id: hal-02264258 \\ https://hal.science/hal-02264258}

Submitted on 6 Aug 2019

HAL is a multi-disciplinary open access archive for the deposit and dissemination of scientific research documents, whether they are published or not. The documents may come from teaching and research institutions in France or abroad, or from public or private research centers.
L'archive ouverte pluridisciplinaire HAL, est destinée au dépôt et à la diffusion de documents scientifiques de niveau recherche, publiés ou non, émanant des établissements d'enseignement et de recherche français ou étrangers, des laboratoires publics ou privés. 


\title{
IShTAR: a test facility to study the interaction between RF wave and edge plasmas
}

\author{
I. Shesterikov, ${ }^{1, a)}$ K. Crombe, ${ }^{2,3}$ A. Kostic, ${ }^{1,2}$ D. A. Sitnikov, ${ }^{4}$ M. Usoltceva, ${ }^{1,2}$ R. Ochoukov, ${ }^{1}$ S. Heuraux, ${ }^{5} \mathrm{~J}$. \\ Moritz, ${ }^{5}$ E. Faudot, ${ }^{5}$ F. Fischer,${ }^{1}$ H. Faugel, ${ }^{1}$ H. Fünfgelder, ${ }^{1}$ G. Siegl,${ }^{1}$ and J.-M. Noterdaeme ${ }^{1,2}$ \\ 1) Max-Planck-Institut für Plasmaphysik, D-85748 Garching, Germany \\ ${ }^{2)}$ Ghent University, Department of Applied Physics, ,B-9000 Gent, Belgium \\ 3) LPP-ERM/KMS, B-1000 Brussels, Belgium \\ ${ }^{4)}$ National Science Center "Kharkov Institute of Physics and Technology", Institute of Plasma Physics, 61108, Kharkov, \\ Ukraine \\ ${ }^{5)}$ Institute Jean Lamour, F-54011 Nancy, France
}

(Dated: 10 July 2019)

Existence of high electric fields near an RF antenna launcher causes a number of parasitic phenomena, such as arcing and impurity release, which seriously deteriorate the performance of an ICRF heating scheme in fusion devices. Limited accessibility of the near antenna region in large-scale fusion experiments significantly complicates the associated experimental studies. The IShTAR (Ion Sheath Test Arrangement) test facility has been developed with the requirement to provide a better accessibility and diagnosability of plasmas in the direct vicinity of an ICRF antenna. The purpose of this work is to give a detailed description on the experimental setup and the available diagnostics. Furthermore the paper will demonstrate the capability of the experiment to study phenomena near an ICRF antenna launcher which are relevant for large-scale fusion ICRH systems.

\section{INTRODUCTION}

The auxiliary heating is the necessary system of any largescale fusion experiment. The Neutral Beam Injection (NBI) and Ion Cyclotron Resonance Heating (ICRH) are considered to be the main heating sources. However, each of them is the subject of its own limitations. NBI for instance suffer from an adverse Cesium injection into the plasma, which limits the steady state operation. Moreover high energetic neutrals (around $\mathrm{MeV}$ ) deposit substantial part of their energy to electrons rather than ions, which also reduces the efficiency.

Launching ICRF waves in fusion plasma is also associated with a number of difficulties occuring not only in the core region but also in the direct vicinity of the antenna strap. The occurence of a high electric field is responsible for arcs and a substantial fraction of energetic particles, accelerated in these fields, which can lead to the errosion of the antenna surface and can cause a substantial release of impurities into the plasma. The mechanisms underlying the formation of such a high electric field are not completely clear nor the role of this field on arcing and sputtering. Several hypotheses have been proposed. Validation of these models on a large-scale fusion experiment is difficult because of a limited accessibility of the antenna near field region. Meanwhile, the scale of the launched RF power limits the choise of suitable diagnostics. Several dedicated facilities have been developed to gain a better insight into the underlying physical processes ${ }^{1,2}$. This work presents the IShTAR test facility, which has been developed to study the physical phenomena in the ICRH antenna near field region ${ }^{3}$. The allocated diagnostic ports and lower level of an applied RF power (since heating is not the goal here) provide good accessibility and diagnosability of the antenna region. In addition to presenting the conceptual design

\footnotetext{
a)Electronic mail: ilys@ipp.mpg.de
}

of this experiment, the paper presents the diversity of available diagnostics, discussing in parallel the associated diagnostic issues. The necessary study on identifying the operating parameters, which resemble the real ICRH antenna near-field conditions for fusion plasmas, is also presented, demonstrating the relevance of this experiment for large-scale ICRH systems.

\section{THE SETUP}

The vacuum vessel of the experiment consist of two modules: the main chamber and the plasma source. The detailed view of the experiment is shown in Fig. 1. The purpose of such a modular configuration is to separate the measurement chamber from the plasma source, thereby improving flexibility and accessibility for the measurements. Each module is equipped with a set of magnetic field coils ( 5 in the plasma source and 2 in the measurement chamber), which are also shown in Fig. 1 (a) and (b). The partially or fully magnetized plasma produced in the source is confined by the magnetic field and flows along magnetic field lines forming a steadystate plasma density spatial profile in the main chamber. The next subsections are devoted to more detailed descriptions of correspondings parts and systems.

\section{A. Plasma source}

The plasma source module is composed of a left-handed half turn helical antenna (shown in the inset in Fig. 1(a)) wrapped around the quartz vacuum vessel and permeated by an approximately uniform magnetic field. The antenna is 64 $\mathrm{cm}$ long and $44 \mathrm{~cm}$ in diameter. It launches fast and slow wave components and has such a helicity that it launches $m=+1$ helicon waves anti-parallel to the $B$ direction and $m=-1$ waves parallel to $B$. The direction of $\vec{B}$ is also shown in the fig- 

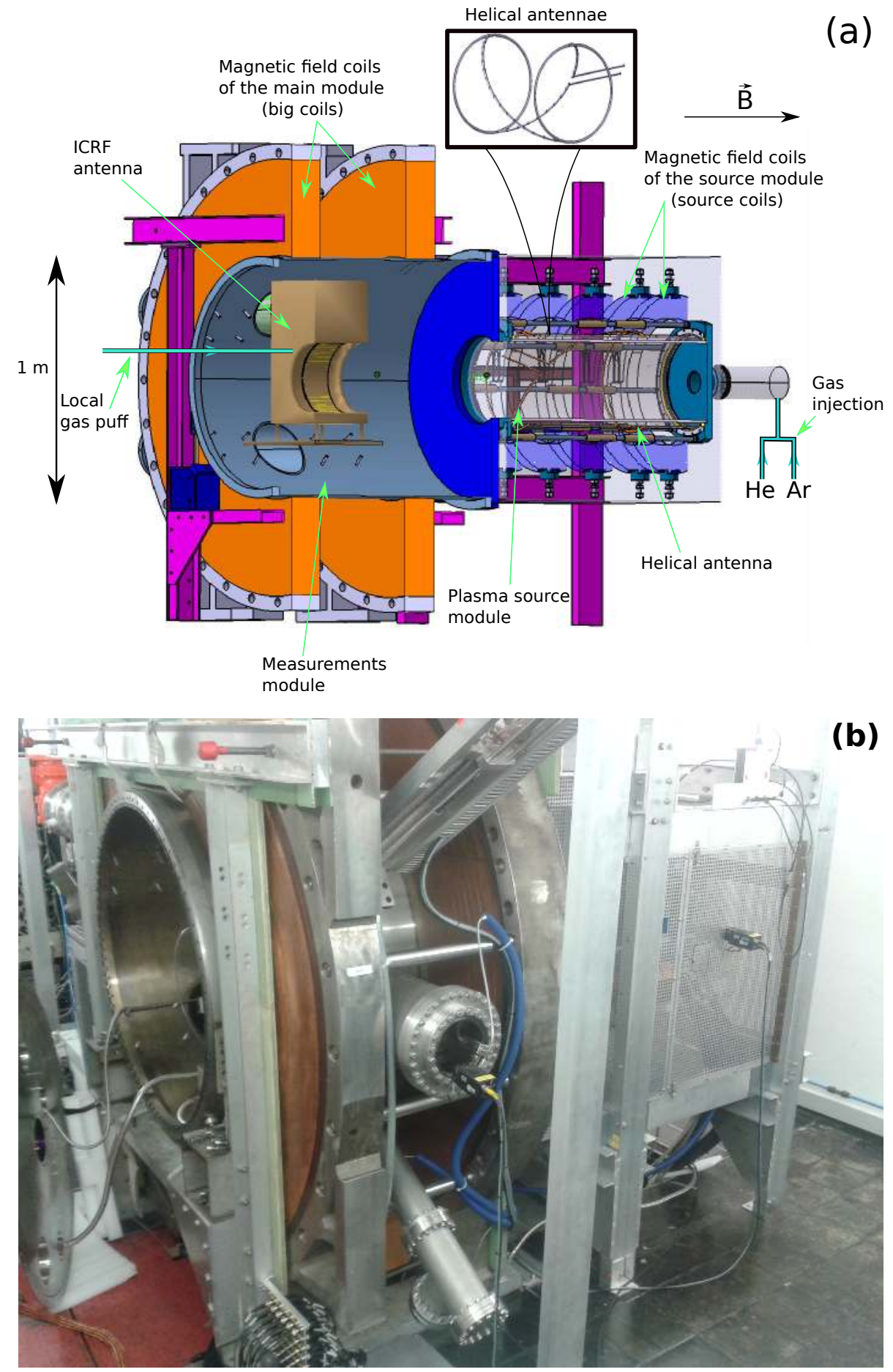

FIG. 1: (a) Cross-sectional view of the entire experimental setup. Key components are indicated. The direction of $\vec{B}$ is also shown by the black arrow. (b) The picture of the experiment.

ure. The mode $m=-1$ is evanescent leaving only the $m=+1$ propagating anti-parallel ${ }^{4-8}$ to $B$, so that the excited RF waves propagate towards the measurement region.

The entire plasma source is shielded by the aluminium Faraday cage, thus protecting the surrounding equipment from electromagnetic interference induced by the RF waves. The available RF amplifier is able to deliver up to $3 \mathrm{~kW}$ of RF power at around $12 \mathrm{MHz}$ with a typical pulse width of $12 \mathrm{~s}$. The forward and reflected power are measured with the directional coupler.

The matching circuit is introduced to match the $50 \Omega$ impedance of the RF amplifier and coaxial cable to the arbitrary impedance of the system "RF antenna + plasma". It is shown in Fig. 2 and presents a so called $\pi$-network. It is 


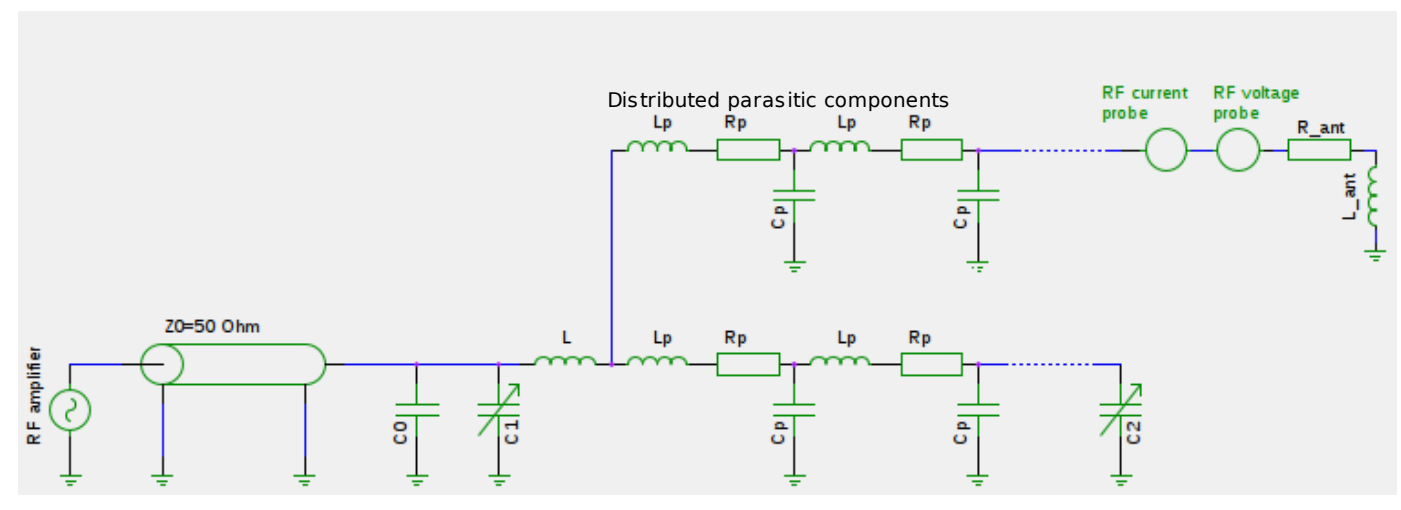

FIG. 2: The equivalent circuit of the matching box for the helical antenna.

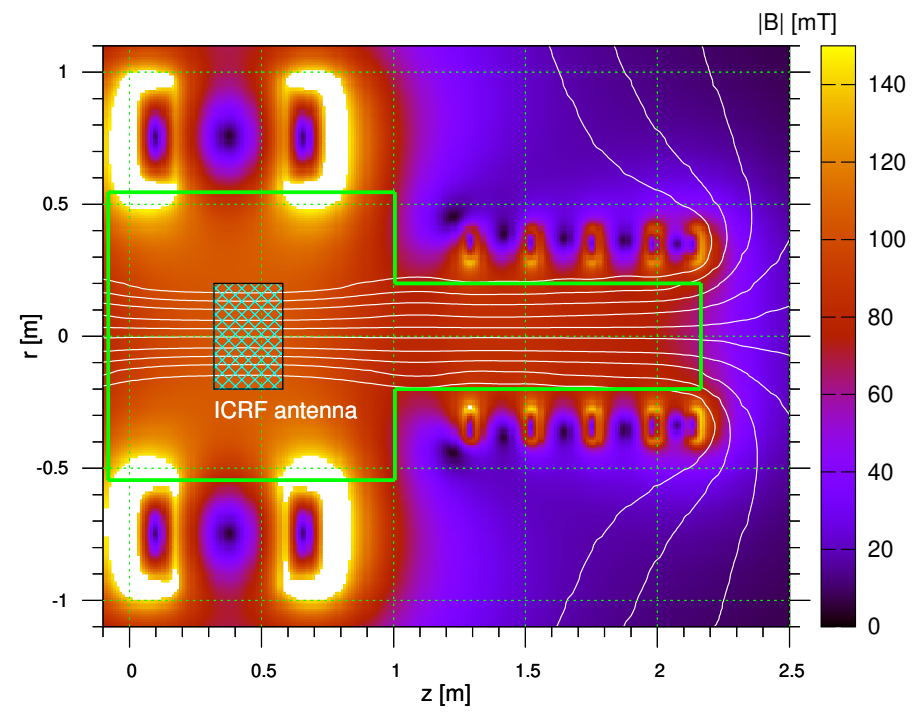

FIG. 3: Example of the magnetic configuration for the distribution of currents in big and source coils $I_{\text {big }}=3000 \mathrm{~A}$ and $I_{\text {source }}=1000 \mathrm{~A}$. The white lines indicate the trajectories of the magnetic field lines while color palitra indicate the magnetic field strength in $\mathrm{mT}$. The green lines show the position of the vacuum vessel wall. The location of the ICRF antenna is also depicted.

composed of a fixed value inductance $L \approx 4.3 \mu H$, one fixed value capacitor $\left(C_{0}=4000 \mathrm{pF}\right)$ and two variable capacitors $\left(C_{1}=247.12 p F-2000 p F, C_{2}=14.05 p F-78.6 p F\right)$. The inductor is the simple 12 turns solenoid, $8 \mathrm{~cm}$ in diameter and $21 \mathrm{~cm}$ in length wound around plastic cilindrical support without ferromagnetic core. Both conductors connecting the inductor $L$, the capacitor $C_{2}$ and the antenna are relatively long $(\approx 50-70 \mathrm{~cm})$, which adds parasitic elements to the ideal $\pi$ network. Moreover the parisitic nature of these components is such that they are distributed (represented as $L_{p}, R_{p}, C_{p}$ ) and in reality cannot be represented as single lumped components. This significantly complicates the mathematical modelling of the equivalent circuit with the aim to find the proper values of $C_{1}$ and $C_{2}$ which match the input impedance of the circuit to the characteristic impedance of the transmission line.
In addition, the matching procedure partially implies the adjustment of the resonance frequency of the entire circuit to the frequency of the RF generator. At the position of this resonance any arbitrary small value of the antenna resistance $R_{\text {ant }}$ could be transformed to the desired $50 \Omega$ However, the shape of the resonance curve is usually narrow. Therefore, in the vicinity of the resonance the input impedance strongly depends on the frequency and a minor shifts of the resonance frequency (for instance due to parasitic impedances ) leads to a significant difference in the input impedance. That is another reason why the mathematical modelling of the entire matching circuit is impractical. In reality the values of $C_{1}$ and $C_{2}$ are established merely empirically. Details on the matching tachnique of the RF antenna to the transmission line could be found in Ref. ${ }^{9}$. The entire matching box is housed in an aluminium case, inside which the RF current transformer and capacitive RF high voltage divider are installed to measure the antenna current and voltage, respectively.

\section{B. Identification of the helicon mode}

The presence of the helicon operation mode has been identified in $\mathrm{Ar}$ plasma by measuring the achievable plasma density $n_{e}$ as a function of the axial magnetic field. The results are shown in Fig. 4. The so-called RF compensated probe ${ }^{8}$ has been positioned on the axis of the source chamber and is biased negatively at $-60 \mathrm{~V}$. The observed $n_{e}(B)$ dependency is linear, which is in qualitative agreement with the general dispersion relation for helicon waves ${ }^{10,11}$ :

$$
n_{e}=\frac{k_{z} k}{e \omega \mu_{0}} B-\frac{m k^{2}}{e^{2} \mu_{0}}
$$

Here $k_{z}$ is the parallel komponent (along $B$ ) of the wave vector $k, e$ is the elementary charge, $\omega$ is the angular frequency of the launched helicon waves and $\mu_{0}$ is the vacuum permeability and $m$ is the electron mass.

The measurements have been done for different neutral $\mathrm{Ar}$ gas pressures in the range $0.9-5.5 \times 10^{-3}$ mbar. In all curves the linear trend lasts only up to a certain threshold $B$ value, above of which the sharp density drop is observed indicating 


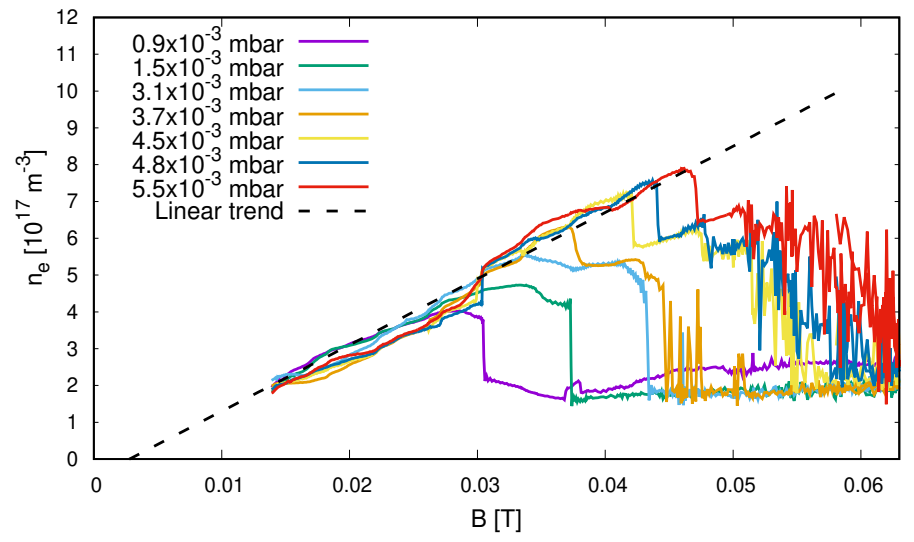

FIG. 4: The dependency of the plasma density vs axial magnetic field in the plasma source module. The observed linear trend and threshold transition are features of the helicon discharge.

some mode transition. For higher values of $p$ such a transition occurs at larger values of $B$, although the linear slopes remains approximately the same. This is also in agreement with the helicon wave dispersion relation in Eq. (1), where the coefficient in front of $B$ does not depend on neutral gas pressure.

The existence of the observed mode transition is another feature of the helicon discharge. Similar observation of such a density peak have been reported previously ${ }^{10,12-15}$ and are associated to a transition from a helicon discharge to a capacitive or inductive discharge ${ }^{16}$.

The physical reason underlying such a transition are not discussed in the literature. The authors propose the following qualitative explanation. The achievable plasma density should satisfy not only the dispersion relation but should also correspond to the energy balance since one can not continue to increase the plasma density merely on account of the magnetic field. It means that the growth of $n_{e}$ with $B$ should be accompanied by the growth of the fraction of RF power absorbed in the plasma. The rest of the power will be absorbed elsewhere in the wall or matching box. Apparently, while rising the $\mathrm{B}$ value, sooner or later the launched RF power is not sufficient to maintain a further growth of $n_{e}$. In this case a further increase in $B$ without a corresponding increase in $n_{e}$ shifts, down the $k_{\|}$cut-off value

$$
k_{\| c u t}=\sqrt{\frac{\omega \omega_{p e}^{2}}{\Omega_{e} c^{2}}}
$$

$k_{\| \text {cut }}$ corresponds to the maximum allowed $k_{\|}$for helicon waves. Sooner or later $k_{\| c u t}$ will be lower than the $k_{\|}$spectrum excited by the antenna. Excitation of Helicon waves in this case will no longer be possible. That corresponds to the observed threshold transition to a much lower densities in Fig. 4. This qualitative and descriptive picture needs to be supported by a more quantitative and detailed analysis. This is outside the scope of this work and will be the subject of further study.

\section{Main vessel}

The cylindrical main vessel is $1.1 \mathrm{~m}$ in diameter and $1 \mathrm{~m}$ long. It is equipped with a positioning system enabling a onedimensional scan in the radial direction with a spatial precision of $\approx 2 \mathrm{~mm}$. Another positioning system permitting a spatial scan along the axial direction is under construction. The antenna is mounted on the vessel wall, as visible in Fig. 1(a). Currently the so-called "fast wave antenna" is installed inside the main module exciting primarily the fast (magnetoacoustic or compressional) plasma wave, although another antenna enabling to launch slow (shearing) wave is planned. It is also considered to install an antenna turnable with respect to the axial magnetic field, thus enabling to launch simultaneously both slow and fast waves, similar to the real ICRH antenna in fusion devices. At the moment up to $1 \mathrm{~kW}$ of RF power is feed to the antenna through the vacuum coaxial feedthrough. However the coaxial transmission line for the ICRF antenna has the capability to launch up to several hundreds $\mathrm{kW}$ of the RF power. The generator enabling to launch such a high power will be available in the near future. Such a high power makes it possible to achieve much higher plasma density in $A r$, but more importantly in $\mathrm{He}$ or deuterium plasmas, thus making the test facility even more relevant for fusion ICRH systems.

\section{Magnetic field strength and topology}

The set of coils for the plasma source (hereinafter referred to as "source coils") is composed of 5 former WEGA stellarator coils. Each coil has an inner radius $R_{\text {in }}=0.285 \mathrm{~m}$, outer radius $R_{\text {out }}=0.395 \mathrm{~m}$, thickness $d=0.05 \mathrm{~m}$ and the total number of turns $N_{\text {turns }}=13$ arranged in a two pancakes. Although developed for a maximal current of $10 \mathrm{kA}$ the present power supply for these coils allows for a continuous current up to 1 $\mathrm{kA}$. Such a current creates in the center of the source module a magnetic field with a strength of $64 \mathrm{mT}$. The set of coils for the main chamber (hereinafter referred to as "big coils") is composed of 2 former W7-A toroidal field coils $\left(R_{i n}=0.55 \mathrm{~m}\right.$, $\left.R_{\text {out }}=0.95 \mathrm{~m}, d=0.183 \mathrm{~m}, \mathrm{~N}_{\text {turns }}=25\right)$ arranged in a configuration similar to Helmholtz coils, i.e. coils are placed at the same distance from each other as their radius. With the available current source they can be supplied with a maximum current of $8 \mathrm{kA}$ for $10 \mathrm{~s}$, although the typically applied current is around $2 k A$. This $2 k A$ current corresponds to the magnetic field on the axis of the main chamber of $68 \mathrm{mT}$. The direction of the magnetic field has been selected to point from the plasma source towards the main chamber, as shown in Fig. 1(a). This direction has been selected taking into account features of the helicon wave propagation, as will be discussed later in this section. The plasma flow from the source to the main chamber can be tailored by varying currents in both sets of coils and thus shaping the plasma profile in the main chamber with the aim to achieve the relatively dense but, on the other hand, not too narrow plasma profile.

The entire magnetic field has been evaluated as a linear superposition of contributions from each coil. The field for each type of coils has been evaluated semi-analytically consider- 
ing each coil as consisting of a number of circular currentcarrying loops of wire with small cross-sections, the contribution of which is then summed up.

The magnetic field lines are sometimes graphically a more representative way to visualize the magnetic field than the vector field. Because of this, it is much more convenient to characterize the magnetic field first in terms of a vector potential $A$ and then to evaluate the magnetic field strength as $B=\nabla \times A$ instead of a direct evaluation of $B$. The point is that the lines of a magnetic field can be evaluated relatively fast and precise as contour-lines of a vector potential, whereas the evaluation from the vector field of $B$, requires costly and imprecise line tracing procedures. The expression for the vector potential of a loop of radius a, carrying a current $I$ in spherical coordinates ( $r, \theta, \phi$ - radial distance $r$, polar angle $\theta$, and azimuthal angle $\phi)$ is given by the following expression:

$$
A_{\phi}(r, \theta)=\frac{\mu_{0} I a}{4 \pi} \int_{0}^{2 \pi} \frac{\cos \phi^{\prime} d \phi^{\prime}}{\sqrt{a^{2}+r^{2}-2 a r \sin \theta \cos \phi^{\prime}}}
$$

The typical magnetic field configuration, which shows the magnetic field line topology and field strength is shown in Fig. 3. It corresponds to currents in source and big coils of $I_{\text {source }}=1000 \mathrm{~A}$ and $I_{\text {big }}=3000 \mathrm{~A}$, respectively. By varying the magnetic field distribution in the big and source coils, one can get either a divergent or a convergent topology with respect to the plasma source, this will significantly affects the plasma transport and flows from the plasma source and, as a consequence, also the radial plasma profile in the main chamber.

\section{E. Gas injection}

Two gas injection systems are implemented at IShTAR. One is the general one, providing the background neutral gas pressure. Pipes and valves of this system are mounted at the plasma source, as shown in Fig. 1(a). He, $\mathrm{Ar}$ or a certain mixture between them can be selected as a working gas on a choice. The necessary mixture can be set varying the mas flow rate at the corresponding mass flow controllers. Both $\mathrm{Ar}$ and $\mathrm{He}$ flow controllers are identical and enable flow rate up to $100 \mathrm{sccm}$ for each gas species. The typical operational gas pressure in the vessel is $\sim(1-2) \times 10^{-3}$ mbar. For $A r$ it corresponds to the $8-16 \mathrm{sccm}$ of flow rate, although for $\mathrm{He}$ the maximum allowable $100 \mathrm{sccm}$ is needed to maintain this pressure. Therefore, installation of the second controller for $\mathrm{He}$ enabling flow rate up to $1000 \mathrm{sccm}$ is currently ongoing.

Another gas injection system is the local gas puff in the vicinity of the ICRF antenna. It is implemented to facilitate the spectroscopic electric field measurements near an ICRF antenna. Local gas puff could potentially improve the emission intensity of the used $\mathrm{He}$ spectral line, which is hardly observable both due to low plasma and neutral gas densities.

\section{F. Control system and database}

The industrial "Siemens Simatic S7" control system is implemented at IShTAR to enable remote operation and setting of almost all key operation parameters, such as gas flow rate, currents in magnetic field coils, RF power in helicon antenna, water cooling. The coil currents on a choice can be either set constant throughout the entire discharge or time trace can be programmed according to a certain assigned scenario. The vacuum tab enables the remote status monitoring and operation of all components of the vacuum system including valves, pumps and gas flow controllers. The safety interlocks system is also implemented within the control system. It also transfers all operation parameters to the data acquisition system to store them in the IShTAR database.

The implemented database presents the individual "*.hdf5" files enumerated according to shot numbers and stored on the hard drive. It is based on the "National Instruments" hardware in connection with the "LabView" software. Each file contains not only data from specific studies recorded with high time resolution $\sim 1 \mathrm{MHz}$, but also general operational parameters like time traces of coil currents, gas pressure, which are sampled with a much slower rate $5 \mathrm{~Hz}$.

\section{G. Typical plasma parameters}

Table I gives an overview of the typical plasma and operational parameters at the IShTAR experiment for different working gases $\mathrm{Ar}$ and $\mathrm{He}$ and experimental conditions relevant for this work.

\section{DIAGNOSTICS}

\section{A. Langmuir probes}

Set of Langmuir probes arranged in arrays are routinely used in the measurements. One array is designed to installed on the positioning system to measure the radial distribution of plasma parameters with a high spatial resolution. An additional axial shaft can be installed on the positioning system to position the probe at a necessary axial location. Another fixed array consisting of 10 probes and covering the radial range from $-20 \mathrm{~cm}$ to $20 \mathrm{~cm}$ can be positioned at any axial position in the vessel to measure the radial distribution of plasma parameters (usually $n_{e}$ and $T_{e}$ ) with a rough resolution of $4.44 \mathrm{~cm}$. Manually moving this array along the axial direction enables a two-dimensional(radial vs axial) scan of plasma parameters. A number of techniques have been developed to adapt the traditional single Langmuir probe for measurements in RF discharges ${ }^{17,18}$.

The technique used in this work refers to the work of Sudit\&Chen ${ }^{17}$ about so-called "RF-compensated" Langmuir probes, although in our experiment it has been implemented in a slightly simplified form. The original concept requires the implementation of additional floating electrode with an collecting area much larger than the primary probe pin. The 
TABLE I: Summary of the typical plasma and operational parameters at the IShTAR experiment for Ar and $H e$ plasmas.

\begin{tabular}{|c|c|c|c|}
\hline & Ar plasma & & He plasma \\
\hline gas pressure $p[$ mbar $]$ & $(1-9) \times 10^{-3}$ & & $10^{-3}$ \\
\hline magnetic field $B[m T]$ & $<150$ & & $<150$ \\
\hline RF power at helicon antenna $P_{\text {helicon }}[k W]$ & $2-3$ & & $2-3$ \\
\hline RF power at ICRF antenna $P_{I C R F}[k W]$ & 1 & & 1 \\
\hline plasma density $n_{e}\left[m^{-3}\right]$ & $<1 \times 10^{18}$ & & $<5 \times 10^{16}$ \\
\hline electron temperature $T_{e}[\mathrm{eV}]$ & 5 & & 10 \\
\hline ion temperature $T_{i}[\mathrm{eV}]$ & room temperature $25 \times 10^{-3}$ & & room temperature $25 \times 10^{-3}$ \\
\hline Debye length $\lambda_{D}[\mu \mathrm{m}]$ & 14 & & 100 \\
\hline electron gyroradius $\rho_{c e}[\mathrm{~mm}]$ & 1 & & 1.3 \\
\hline ion sound radius $\rho_{s i}[\mathrm{~mm}]$ & 20 & & 4 \\
\hline electron cyclotron frequency $f_{c e}[\mathrm{GHz}]$ & & 2.8 & \\
\hline ion cyclotron frequency $f_{c i}[\mathrm{kHz}]$ & 39 & & 795 \\
\hline electron-ion collision frequency $v_{e i}$ & $2.6 \mathrm{MHz}$ & & $45 \mathrm{kHz}$ \\
\hline electron-total collision frequency $v_{e n}[\mathrm{MHz}]$ & 8.2 & & 3.2 \\
\hline ion-neutral collision frequency $v_{i n}[\mathrm{kHz}]$ & 17 & & - \\
\hline
\end{tabular}

detailed analysis show that the presence of the secondary electrode is not really necessary if the primary pin has a relatively large collecting area. $C_{s h}$ and $R_{s h}$ are the sheath capacitance and sheath resistance (of the probe pin), which give rise to the sheath impedance $Z_{s h}$. The sheaths impedance of the auxiliary electrode is $Z_{x}$. In the original concept $Z_{x}$ is added in parallel to $Z_{s h}$ and forms a new equivalent impedance $Z_{s h \| x}$. The purpose of the auxiliary electrode is to reduce the effective sheath impedance $Z_{s h \| x}$. A RF choke, self-resonant at the $\mathrm{RF}$ frequency, is placed close to the probe pin to force it to float together with plasma potential fluctuations. We denote $Z_{c h}$ as the impedance of the RF choke. The choke and sheath form a voltage divider for the induced plasma potential. The following condition must be satisfied in order for the scheme to work $^{8}$ :

$$
Z_{c h} \gg Z_{s h \| x}\left(\frac{e V_{r f}}{k T_{e}}-1\right),
$$

where $V_{r f}$ denotes the amplitude of the RF induced plasma potential fluctuations. Typically in IShTAR conditions $V_{r f}$ amplitude does not grow larger than $70 \mathrm{~V}$. This condition implies that the induced RF voltage drops not at the RF sheath but on the entire probe pin. In this case the sheath voltage drop, which determines the current flowing through the probe, does not fluctuate with the RF frequency and is determined by the difference $V_{D C}-\phi_{p}\left(V_{D C}\right.$ is the applied DC potential of the voltage source and $\phi_{p}$ is the mean value of the plasma potential). The auxiliary electrode helps to reduce $Z_{s h \| x}$ in order to satisfy the above relation.

The introduced simplification is that the auxiliary electrode has been omitted in our design. Indeed, the presence of an auxiliary electrode is not really necessary as soon as the collecting area of the main probe pin is large enough to satisfy the Rel. 4. The sheath resistance is given as follows ${ }^{8}$

$$
R_{s h}=\frac{4 \lambda_{D}^{2}}{\varepsilon_{0} A_{p} v_{s}}
$$

where $\varepsilon_{0}$ is the vacuum permittivity $\lambda_{D}$ is the Debye length and $A_{p}$ is the probe surface area (for our cylindrical probe pin: length $l=10 \mathrm{~mm}$, diameter $d=1 \mathrm{~mm}$ and surface $A_{p}=l \pi d^{2} / 4 \approx 62 \mathrm{~mm}^{2}$ ) and $v_{s}$ is the sound speed $v_{s}=\frac{\sqrt{k T_{e}}}{M}$. For typical IShTAR plasmas $\left(n_{e} \sim 1 \times 10^{18} \mathrm{~m}^{-3}\right.$ and $\left.T_{e} \sim 6 \mathrm{eV}\right)$ $\lambda_{D} \approx 18 \mu \mathrm{m}$. The sheath capacitance is estimated as follows ${ }^{19}$

$$
C_{s h} \sim \frac{\varepsilon_{0} A_{p}}{\lambda_{D}}
$$

and the capacitive contribution ${ }^{8}$

$$
Z_{C s h} \sim \frac{1}{j \omega C_{s h}} .
$$

The sheath impedance is then ${ }^{20}$

$$
Z_{s h}=\frac{R_{s h} Z_{C s h}}{R_{s h}+Z_{C s h}}
$$

For typical IShTAR plasmas and at a routinely used helicon frequency of $12 \mathrm{MHz}$, the typical sheath impedance $Z_{s h} \approx$ $438 \Omega$. This impedance, multiplied by the factor $\left(\frac{e V_{r f}}{k T_{e}}-1\right) \sim$ 10 gives the right hand side of the condition given in relation 4 , i.e. approximately $5 k \Omega$. The impedance of the RF choke at the resonance frequency is about $20-25 k \Omega$, which is $4-5$ times higher than the right hand side of relation 4 .

Several tipps can be given in the design of the corresponding RF choke. In the original work of Sudit\&Chen ${ }^{17}$ a conventional inductance whose resonance frequency coincides with the RF generator frequency is proposed. It provides a compact and easily implementable solution. The variety in the nomenclature of commercially available inductances always allows to find a component with a suitable resonance frequency. The conventional inductor, however, has a ferromagnetic core, which allows to reduce the number of windings and obey compactness. However, the obvious concern in 
using ferromagnetic materials in the magnetic field is the saturation. The saturation level of ferrites is $0.2-0.5 T$, which is comparable to magnetic field values typically used in our experiment and other helicon discharges. However already at much lower values of $B$ a small deviation in the inductivity, leading to small shifts of the resonance frequency, will be noticable. Note that the resonance curve of a RF choke must be very narrow (i.e. has a high $Q$ value) and a small deviations in resonance frequency leads to a noticable difference in the impedance at a given fixed frequency: that is a typical behaviour of resonant circuits.

(R)Murata $2 \mathrm{~mm}$ width SMD (Surface-mount device) inductors, such for instance as "LQW2UAS4R7J00"(4.7 $\mu H)$, with non-magnetic core material, which is not disturbed by the external magnetic field, has been used in our work. The self-resonance frequency of this inductor lies in the range of $200-300 \mathrm{MHz}$. Therefore, the corresponding SMD capacitance (also $2 \mathrm{~mm}$ width) is soldered in parallel with the inductor in order to shift the resonance frequency down to the helicon antenna frequency.

\section{B. Magnetic probes}

Magnetic probes are designed to detect simultaneously all three components of the RF magnetic field. Therefore they contain three loops oriented mutually perpendicular to each other. The structure of the probe is shown in Fig. 5. Loops are fixed on the walls of the square ceramic holder (10 $\mathrm{mm}$ long), which ensures good mutual perpendicularity in the orientation and protect the loops from strong heat load from plasma. The holder is produced by cutting a long square ceramic tube into short lengths. The special high temperature ceramic glue (type "618-N" from (RCeramabond), which can sustain up to 1650 ${ }^{\circ} \mathrm{C}$, is used to fix the loops to the inner wall of the holder. One should consider several concerns when designing such a magnetic probe to measure the RF signal.

Signal lines from each loop are connected to the signal detector, which has $R=50 \Omega$ input impedance in order to match with the $50 \Omega$ characteristic impedance of a transmission line. This means that the external RF magnetic field $\widetilde{B}_{R F}$ induces in the loops not only the voltage signal $\widetilde{U}_{\text {loop }}$ but also the current $\widetilde{I}_{\text {loop }}=\frac{\widetilde{U}_{\text {loop }}}{R}$. This problem is absent in measuring low frequency magnetic field signals (below $1 \mathrm{MHz}$ ) since matching is not an issue at this frequency range. The current $\widetilde{I}_{\text {loop }}$, on the other hand, will induce in the loop a corresponding response magnetic field of

$$
\widetilde{B}_{\text {resp }}=\mu \frac{N}{l} \widetilde{I}_{\text {loop }}
$$

where $N$ and $l$ are number of turns and the loop length, respectively.

The current $\widetilde{I}_{\text {loop }}$, in turn, depends on the induced voltage

$$
\widetilde{U}_{\text {loop }}=N A \widetilde{B}_{R F} \omega
$$

where $A$ is the loop area and $\omega$ is the RF frequency. The response magnetic field $\widetilde{B}_{\text {resp }}$ is then

$$
\widetilde{B}_{r e s p}=\mu \frac{N}{l} \frac{N A \widetilde{B}_{R F} \omega}{R}=\frac{L \widetilde{B}_{R F} \omega}{R},
$$

where $L$ denotes the inductance of the solenoid

$$
L=\frac{\mu N^{2} A}{l} .
$$

The response field should not perturb the measured RF field $\widetilde{B}_{\text {resp }} \ll \widetilde{B}_{R F}$, which imposes the following limitation on the design of loops.

$$
L \omega \ll R .
$$

In addition, the loop inductane $L$ together with the resistance $R$ form a low-pass filter with a cutoff frequency $f_{c}=\frac{R}{2 \pi L}$. The frequency $f_{c}$ must be well above the RF frequency $\frac{\omega}{2 \pi}$ in order to minimise a signal attenuation. This coincidentally gives the same condition as the one in Eq.13.

Eq.13 imposes to minimise $N, A$ and maximise $l$. This, however, also reduces the measured signal $\widetilde{U}_{\text {loop }}$. Therefore the requirement in Eq.13 should be satisfied but not too strongly, i.e.

$$
L \omega<R
$$

The developed magnetic loops typically have radius $3 \mathrm{~mm}$ $\left(A \approx 29 \mathrm{~mm}^{2}\right), N=3$ and length $l \approx 2 \mathrm{~mm}$. At the routinely used frequency $12 \mathrm{MHz}$ this gives $L \omega \approx 12 \Omega$, which is less than $50 \Omega$.

Another issue related to the development of an magnetic probes for RF signals is the electrostatic pick up rejection, i.e. the measured signal should be induced exceptionally by the surrounding magnetic field and not by the electric field picked up capacitively from the plasma potential fluctuations. Several techniques are used to reject the electrostatic pick up:

- electrostatic screen with slits correspondingly made to prevent a RF current flowing on the screen surface

- electrostatic decoupling the magnetic probe and signal detector through an isolation RF transformer

- an auxiliary reference electrode to pick up merely an electrostatic signal for further processing

Isolation RF transformers ( $\mathrm{Q}$ Mini-Circuits, model "MCL703") are used in our work to reject electrostatic signals. The feature of the electrostatic pick up is that it is common mode signal, i.e. it is equal on both contacts of the loop, while the induced magnetic signal is purely differential. The beneficial feature of the transformer is that it transmits only the differencial component of the signal omitting the common mode part.

One of the conceptual difficulty of IShTAR magnetic probe measurements is the small difference between the helicon frequency $12 \mathrm{MHz}$ and the routinely used frequency of an ICRF 


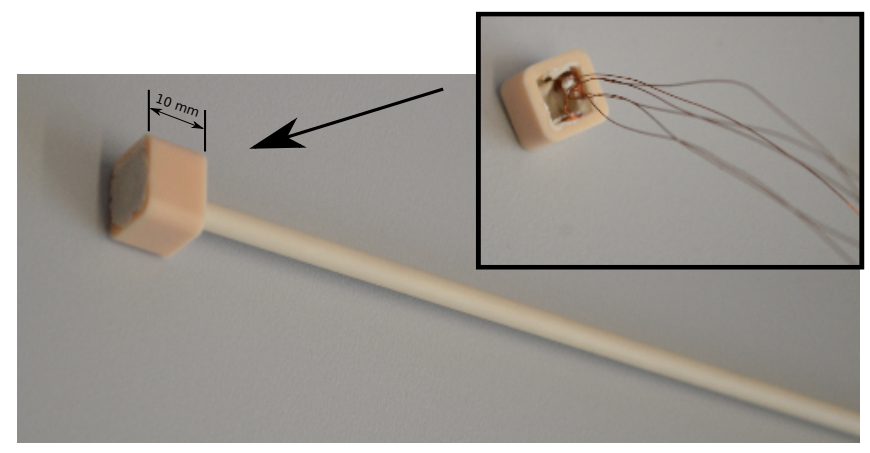

FIG. 5: The picture of the developed three-dimensional magnetic probe.

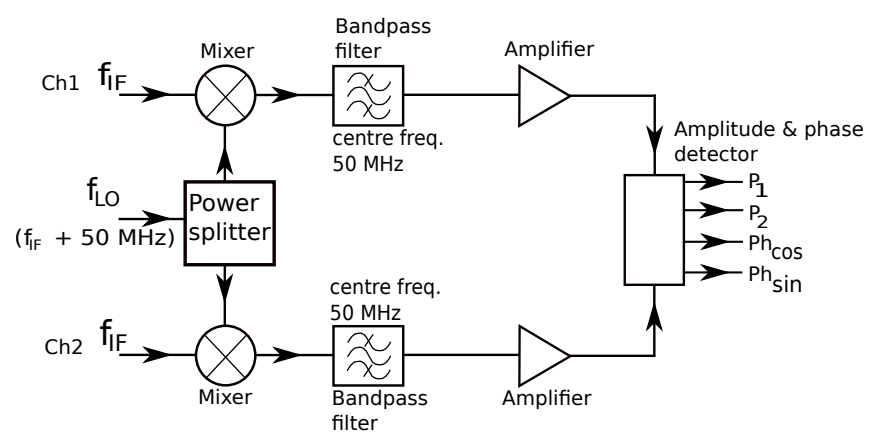

FIG. 6: The selective RF signal detector used for magnetic probe measurements. The concept is based on the superheterodyne receiver scheme. The advantages are easier frequency tuning and high frequency selectivity due to a narrow bandwidth of the crystal filters.

antenna $\sim 6 \mathrm{MHz}$. The so called superheterodyne receiver concept has been applied in order to decouple these two frequencies in the measured signals. Two crucial advantages of this concept determine our choise: the detector can be tuned to a certain desired frequency of interest, which, in the case of the IShTAR detector, could be any frequency in the range from $1 \mathrm{MHz}$ to $20 \mathrm{MHz}$; it enables a high frequency selectivity due to narrowband crystal filters or similar technologies used in the design.

The conceptual design of the magnetic probe detector circuit used in the IShTAR experiment is shown in Fig. 6. Each detector is designed for two input channels (designated as $C h 1$ and $C h 2$ ), the output provides correspondingly the RF power amplitude for each input channel $\left(P_{1}\right.$ and $\left.P_{2}\right)$ and the phase difference between them measured according to the quadrature detection scheme $\left(P h_{\text {cos }}, P h_{\text {sin }}\right)$. An additional external local oscillator input signal (frequency $f_{L O}$ ) is needed for the implementation of the "superheterodyne" concept. In the initial stage the heterodyne detection is applied to two input channels separately, before the amplitude and phase shift between signals is measured by the "Amplitude \& phase detector". The "superheterodyne" detection has the following structure: The existing high- $Q$ bandpass filters, which can give very narrowband filtering, are typically crystal filters or similar technologies and are manufactured only for certain fixed center fre- quencies (i.e. not tunable). The idea is to shift the analyzed frequency to a center frequency of a crystal filter, which allows narrowband filtering (the bandwidth of filters used in our design is $80 \mathrm{kHz}$.). This is achieved using the signal multiplication (or mixing) with another local oscillator signal $\left(f_{L O}\right)$, creating a pair of sinusoidal signals at frequencies $f_{\text {in }} \pm f_{L O}$. The multiplier is called a Mixer (also indicated in the figure), and the frequency $f_{L O}$ is choosen such that one of the mixing products falls into the frequency band of the crystal filter. All other products will be eliminated. The amplifier stage is required to compensate the unavoidable signal attenuation introduced by the mixing and filtering stages. We refer the interested reader to Refs. ${ }^{21,22}$ for more information about this measurement technique. The following components are used in our design: Mixer - "SRA-1" from (RMini-Circuits, Bandpass filter - "MQF 50.0-8000/06" from $囚$ Vectron, Amplifier - "THS 9001" from @Texas Instruments. An RF voltage amplitude and phase difference are measured using "AD8362" and "AD8302" from $§$ Analog Devices, respectively.

\section{Spectroscopic diagnostics}

The optical emission spectroscopy is the main diagnostic tool to measure the electric field in front of the ICRF antenna at IShTAR. Spectroscopy on IShTAR is based on several diagnostic techniques. First, the spectra recorded with the wideband spectrometer are used to monitor the atomic species of the working gas and the impurities. The narrow-band highresolution monochromator is used to assess the Stark shifts of the lines of excited neutral helium atoms, thereby evaluating the electric field vector in the presence of the background stationary magnetic field ${ }^{23}$.

For recording wide-band spectra "AvaSpec-ULS3648 Starline" spectrometer from $囚$ Avantes ${ }^{24}$ is used. This portable, plug-and-play device is of a "Czerny-Turner" optical mount, with a plane grating with 300 lines $/ \mathrm{mm}$ line density and the wavelength range from $200-1100 \mathrm{~nm}$. The system, with a non-fixed slit size, provides spectral resolution of as low as $0.7 \mathrm{~nm}$.

To be able to follow the shape of the recorded spectral line ${ }^{23}$, we routinely use "Shamrock 750" monochromator from $\AA A N D O R$, with a focal length of approximately $738 \mathrm{~mm}$, aperture of $F / 9.7$, and a detector from $\AA$ ANDOR intensifier camera series "IStar 334T", which provides highresolution, high-sensitivity and fast acquisition rates.

The monochromator, also in a "Czerny-Turner" optical mount, is equipped with three interchangeable gratings $600 \mathrm{l} / \mathrm{mm}$, blaze $500 \mathrm{~nm}$; $2400 \mathrm{l} / \mathrm{mm}$, holographic optimized for $190-800 \mathrm{~nm}$; and a grating with line density of $3600 \mathrm{l} / \mathrm{mm}$, holographic optimized for $180-500 \mathrm{~nm}$ range.

Spectrosocopic lines of sight on IShTAR are fixed on three locations, nevertheless are easily relocated. The are shown in red solid lines in Fig. 7. The light is coupled to any of the spectrometers via the optical fibers (protected by a chromeplated brass jacket). The holders for the fibers and the collimating lenses are located on the mid-plane port in the main vessel, the helicon source and the third one is installed in- 


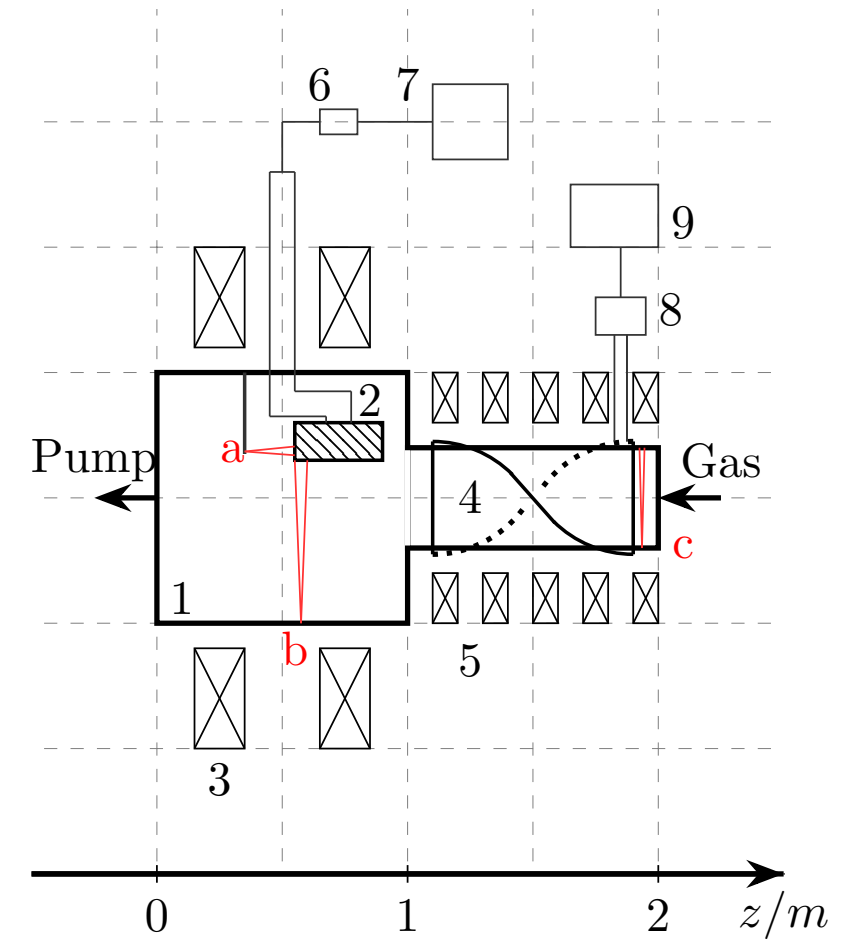

FIG. 7: Schematic representation of IShTAR: 1-main chamber; 2-single strap ICRF antenna; 3-magnetic field coils (big coils); 4-helicon plasma source; 5-magnetic field coils (source coils); 6-autotuner; 7-ICRF generator;

8-matching system; and 9-RF generator; (a) and (b) show the spectrosocopic lines of sight focused on the ICRF antenna edge, where electric field value is considered to be the highest; (c) supplementary line of sight (not used regularly).

side of the main vacuum vessel on the holder fastened close to the leading edge of the ICRF antenna box to measure the near-antenna electric field. More details about spectroscopic diagnostic tools available at IShTAR experiment can be found in Ref. ${ }^{23}$

\section{THE MACHINE CAPABILITY STUDY}

A systematic study of the propagation of waves launched by the ICRF antenna requires a detailed study of the ability to create the required profile of the refraction coefficient $N$. The refraction coefficient in general is a nonlinear function of plasma density and magnetic field, as will be discussed later in this work. Moreover, variations in magnetic field directly affect the plasma density profile due to a number of mechanisms which will also be discussed later. Therefore, as a first step towards the machine capability study, one requires a preliminary investigation of the ability to achieve the necessary plasma density radial profile $n_{e}(r)$ in front of the ICRF antenna for different axial magnetic field values of interest. The required radial profile of $N$ will then be evaluated in a second step, using the calculated $B$ and measured $n_{e}(r)$ as input parameters.

\section{A. Plasma density profile for different magnetic field values}

The first step in the study of the machine capability implies a systematic measurements and storage of plasma density radial profiles as a function of the value of currents in both sets of coils and other various combinations of operation parameters (neutral gas pressure, gas type, helicon antenna power). The aim is to form a certain database of different operation configurations. Such a database contains all relevant information about the operation parameters (magnetic field, neutral gas pressure, gas type, helicon antenna power) needed to create a certain wave propagation scheme. We do not aim to present the entire database within the frame of this limited work and only some representative results will be considered. For example, only results corresponding to an $\mathrm{Ar}$ plasma will be discussed since Ar is used at IShTAR more commonly than Helium. Even though Helium plasma might be more relevant for fusion studies in terms of the ion mass, the achieved plasma density in front of the antenna is too low $\left(<2 \times 10^{16} \mathrm{~m}^{-3}\right)$ to resemble the SOL plasma in tokamak. Meanwhile, a hollow plasma density radial profile in Helium plasma also does not correspond to the real density profile in the tokamak SOL A pure Helium plasma is used at IShTAR primarily for development and commissioning of an optical spectroscopy system to measure the electric field in front of the ICRF antenna. For the same purpose Helium is used sometimes in small amounts as an additive specie to Ar plasma. This, however, is an exceptional case. Only one gas specie is usually used for simplicity since wave propagation in multispieces plasma presents another degree of complexity and will not be considered in this paper. The emphasis will be also done on the governing role of the magnetic field and its topology (i.e. distribution of currents in both sets of coils) since it determines the wave propagation both directly (through magnetic field strength) and indirectly (affecting plasma density profiles).

To simplify the preparation of such a database, the entire radial profile has been measured using a developed Langmuir probe array consisting of 10 probes covering a radial range $-20 \ldots .20 \mathrm{~cm}$, as has been discussed in Sec.III A. Each probe in an array was compensated for a particular frequency of a Helicon antenna $12.04 \mathrm{MHz}$. In addition to traditional parameters, like applied Helicon antenna power or neutral gas pressure, the plasma profile is strongly sensitive to the value of currents in both sets of coils, i.e. to the magnetic field distribution across the experiment. In addition, in comparison to gas pressure and RF power, which affect the plasma density monotonically and mainly quantitatively (i.e. the stronger the RF power the higher density), the magnetic field distribution changes plasma profile non-linearly and qualitatively. There are a number of ways on how the magnetic field distribution can affect the particle transport and Helicon waves propagation from the plasma source towards an ICRF antenna, including

- Simple magnetic mapping, assuming that the field-lines 

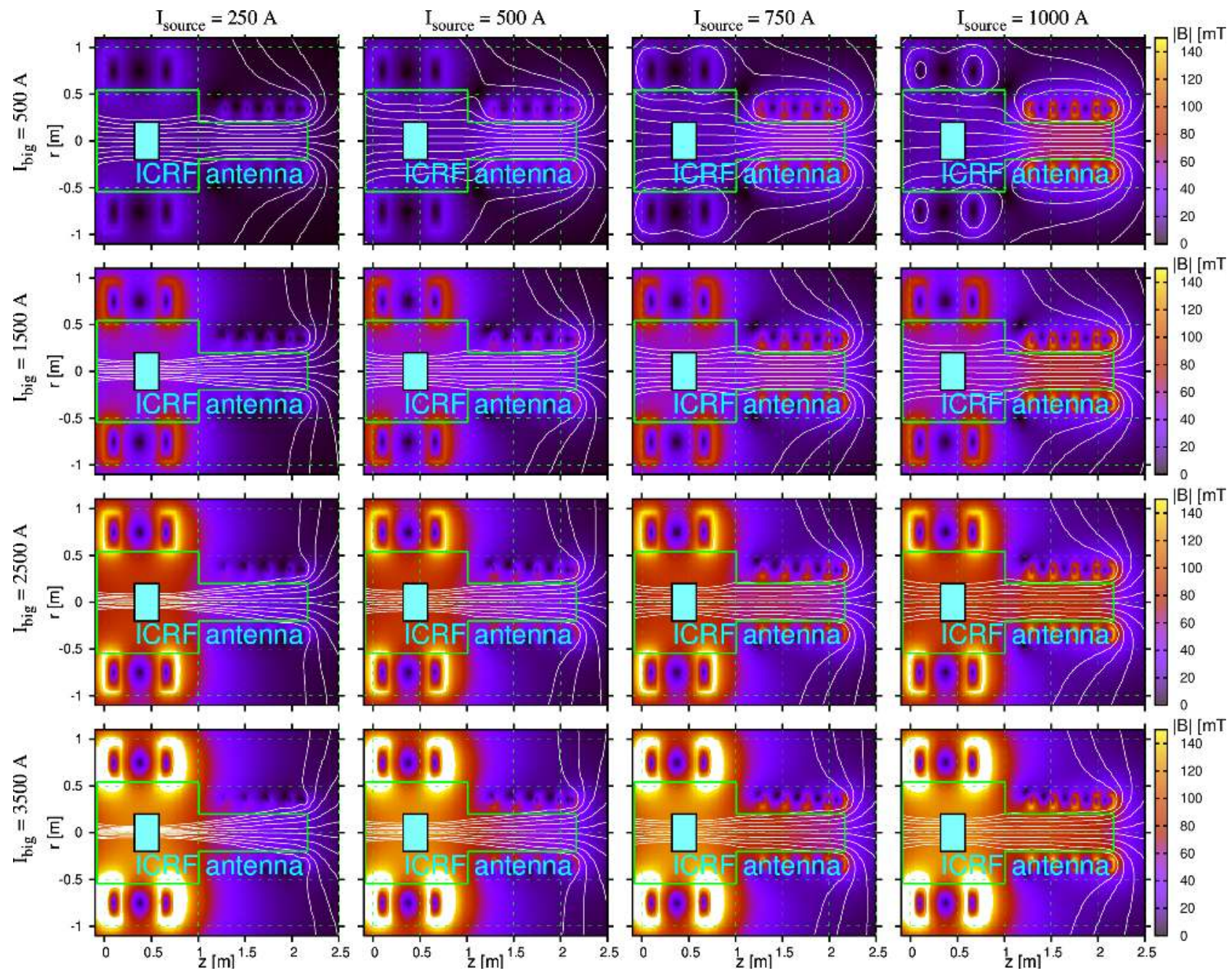

FIG. 8: Magnetic field strength (color palitra) and topology (white lines) across the IShTAR experiment. Each row corresponds to the fixed value of $I_{b i g}$, which changes from $500 \mathrm{~A}$ to $3500 \mathrm{~A}$ from the top to the bottom. Each column corresponds to a certain value of $I_{\text {source }}$, which is also indicated on the top of each column. Green solid lines indicate the position of the vacuum-to-air interface and essentially includes both the plasma source and the main module. The location of the ICRF antenna is shown as a hatched area.

are frozen into the plasma. The plasma frozen in the magnetic field expands or compresses together with the flux tube, leading to the expansion or compression of the plasma profile depending on the divergence or convergence of the magnetic topology outwards from the plasma source.

- The plasma diamagnetism, always repelling plasma towards the region of a low magnetic field, also affects transport depending on the divergence/convergence of the field topology.

- The poloidal plasma currents of different nature, such for instance as a diamagnetic current or $E \times B$ current (if ions are nonmagnetised or not fully magnetised, which is the case for IShTAR $\left(v_{i n} \sim f_{c i}\right.$, see Tab.I $\left.)\right)$, could also lead to the plasma expulsion from the region of an unhomogeneous magnetic field. This mechanism, for exam- ple, forms the basis for magnetic nozzles used in plasma propulsion systems ${ }^{25}$.

- The magnetic field convergence/divergence also affects the Helicon waves upstream and downstream propagation, as has been stated in Ref. ${ }^{11}$.

As a results, the dependency of the plasma profile on the magnetic field presents most interest for a machine capability study. Such a dependency has been experimentally studied. The required magnetic field configuration can be set by finding a proper point in the parameter space $\left(I_{b i g}, I_{\text {source }}\right)$. At first, in Fig. 8 we present for reference some typical magnetic field configurations which are relevant for this study. The magnetic field has been calculated according to the approach described earlier in Sec. II D. Each row corresponds to the fixed value of $I_{b i g}$, which changes from $500 A$ to $3500 A$ from the top to the bottom. Each column corresponds to a certain value of $I_{\text {source }}$, 


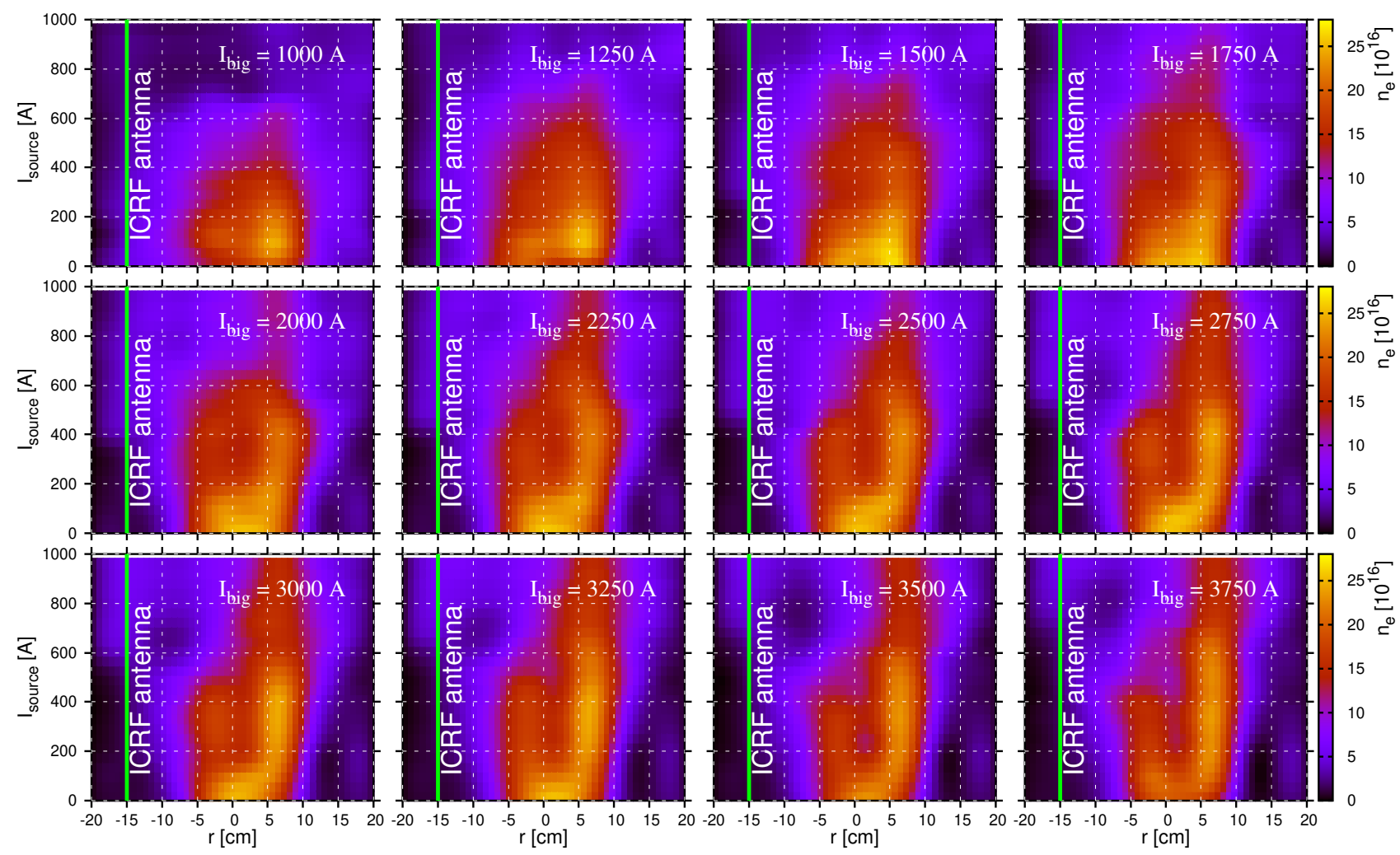

FIG. 9: Plasma density radial profile as a function of $I_{\text {source }}$ for different values of $I_{b i g}$. The radial position of the ICRF antenna is specified with the green vertical line. Corresponding values of $I_{b i g}$ are specified within each plot. The higher $I_{b i g}$ the larger $I_{\text {source }}$ is needed to expand the plasma profile in order to increase the edge plasma density. This trend is in agreement with the simple concept of magnetic mapping.

which is also indicated on the top of the figure.

Only some selected results are presented here, which represent the main features of the magnetic topology. Looking outwards from the plasma source in the direction of the ICRF antenna, the field topology can be converging or diverging, depending on $I_{b i g}$ and $I_{\text {source }}$ values. For instance, for a relatively small $I_{b i g}$ values of $500 A$, the field topology is mostly diverging, except for a very small $I_{\text {source }}$ values of $250 \mathrm{~A}$, when magnetic field lines are almost straight. For an intermediate $I_{\text {big }}$ currents around $2500 \mathrm{~A}$, the straight topology is achieved naturally for higher $I_{\text {source }}$ around $1000 \mathrm{~A}$. For a maximum $I_{b i g}=3500 \mathrm{~A}$, the field is mostly converging and only at $I_{\text {source }}=1000 A$ the nearly straight field lines are achieved. These results give a general qualitative impression about an influence of the field topology on the plasma flow from the source chamber.

The corresponding measurements of plasma density for different magnetic configurations require to explore the whole $I_{\text {big }}, I_{\text {source }}$ parameter space, which could be time consuming. The IShTAR experiment has an seldom feature to speed up these measurements. The power supplies for both sets of coils have a programmable current output. It allows to sweep at least one parameter (either $I_{\text {big }}$ or $I_{\text {source }}$ ) within one discharge throughout the whole range determined by the power supply. This feature has been used in our study. In each discharge the current $I_{\text {big }}$ is kept constant, while current $I_{\text {source }}$ are ramped up linearly from 0 to its maximum value of 1000 A. By varying the current $I_{b i g}$ from shot to shot, one can systematically explore the entire parameter space. The corresponding plasma profiles in front of the ICRF antenna have been measured in Ar plasma and are presented in Fig. 9. Each plot in Fig. 9 represents a reaction of the plasma density radial profile (horisontal axis) on the change of $I_{\text {source }}$ (vertical axis). The results are presented for $I_{b i g}$ values in the range from $1000 \mathrm{~A}$ to $3750 A$ with the step of $250 A$, thus completely covering the whole practically interested parameter space. Each plot corresponds to a fixed value of $I_{b i g}$ which is specified in plots with a white label. The green vertical line indicates the radial position of the ICRF antenna $(\approx-15 \mathrm{~cm})$. Approximately $2 \mathrm{~kW}$ of RF power was applied to the helical antenna. The neutral gas pressure of 1 mbar was maintained throughout the measurements.

Following the results in Fig. 8 the magnetic mapping representation leads to a broadening of the plasma profile when $I_{\text {source }}$ increases, increasing the density at the edge and correspondingly reducing it in the center. Apparently, the measured results in Fig. 9 indeed show such a broadening for almost all presented $I_{b i g}$ values. For instance, for results corresponding to $I_{\text {big }}=1750 \mathrm{~A}$ a central density gradually decreases while the edge density becomes higher with increasing $I_{\text {source }}$. A relatively dense plasma in the vicinity of antenna is achievable for $I_{\text {source }}>300 \mathrm{~A}$. The corresponding edge plasma den- 


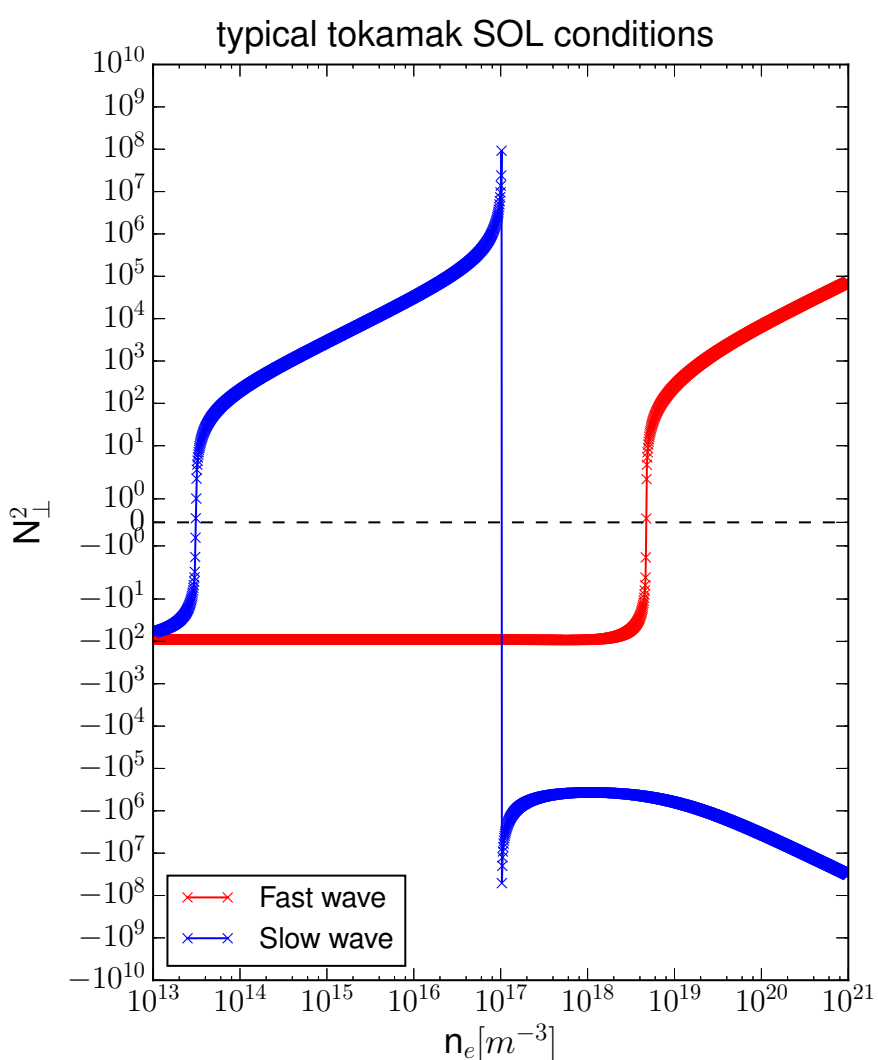

FIG. 10: The perpendicular component of the refraction coefficient in magnetised plasma as a function of the plasma density for deuterium plasma. The wave frequency $\omega=50 \mathrm{MHz}$ and magnetic field strength $B=2 T$ are chosen similar to typical tokamak SOL plasma conditions. Results correspond to an arbitrary value $k_{\|}=10 \mathrm{~m}^{-1}$. Two presented dependencies describe slow and fast wave branches of plasma waves. The singularity for the slow wave around $n_{e} \approx 1 \times 10^{17} \mathrm{~m}^{-3}$ corresponds to the onset of the lower-hybrid resonance.

sity is of the order of $5 \times 10^{16} \mathrm{~m}^{-3}$. For $I_{\text {big }}=2750 \mathrm{~A}$, a dense edge plasma is achieved already for higher $I_{\text {source }}$ values $I_{\text {source }}>600 \mathrm{~A}$.

This trend is noticable throughout all plots: the higher $I_{\text {big }}$ the larger $I_{\text {source }}$ is needed to broaden the plasma profile in order to increase the edge plasma density. This is qualitatively in agreement with the simple concept of magnetic mapping, i.e. plasma expansion together with the expansion of the flux tube. The maximal edge densities achieved by varying $I_{\text {source }}$ do not vary significantly for different plots and remain in the range of $(6-7) \times 10^{16} \mathrm{~m}^{-3}$.

Based on these results one cannot state which configuration presents the most practical interest for ICRF antenna relevant studies since not only the plasma density but also the magnetic field are important quantities in the dispersion relation for plasma waves. From that point of view, the different plots presented in Fig. 9 are not comparable. The purpose of these results is to prepare the background for further analysis

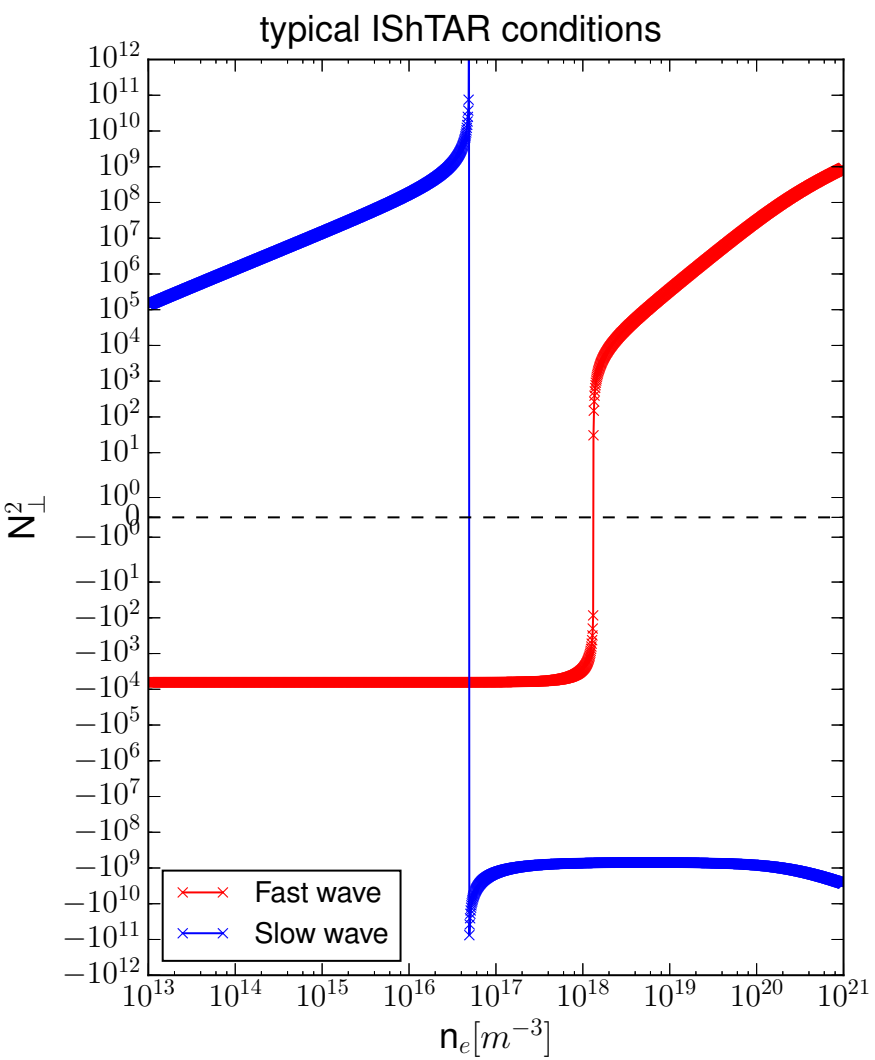

FIG. 11: The perpendicular component of the refraction coefficient in magnetised plasma as a function of the plasma density for $A r$ plasma. The wave frequency $\omega=6 \mathrm{MHz}$ and magnetic field strength $B=0.1 T$ are chosen similar to those in the IShTAR experiment. Results correspond to an arbitrary value $k_{\|}=10 \mathrm{~m}^{-1}$. Two presented dependencies describe

slow and fast wave branches of plasma waves. The singularity for the slow wave around $n_{e} \approx 5 \times 10^{16} \mathrm{~m}^{-3}$ corresponds to the onset of the lower-hybrid resonance.

of wave propagation in terms of the refraction coefficient.

\section{B. Wave propagation}

The description of the propagation of electromagnetic waves in a magnetized plasma is based on the analysis of the dispersion equation. The general consideration in the presence of collisions, thermal motion and spatial density gradients is very complex. Therefore we limit our consideration with the case of a cold collisionless plasma. The wave equation and a corresponding dispersion relation for a cold magnetized plasma has been considered in a number of works ${ }^{26,27}$ although the description primarily used in the ICRF community is based on the so-called "Stix notations"28,29. We will consider the general case of an arbitrary incidence angle $\theta$ between the wave vector $k$ and a magnetic field $B$. The corresponding dispersion relation, which determine the existence of a meaningful solution of the wave equation is a 

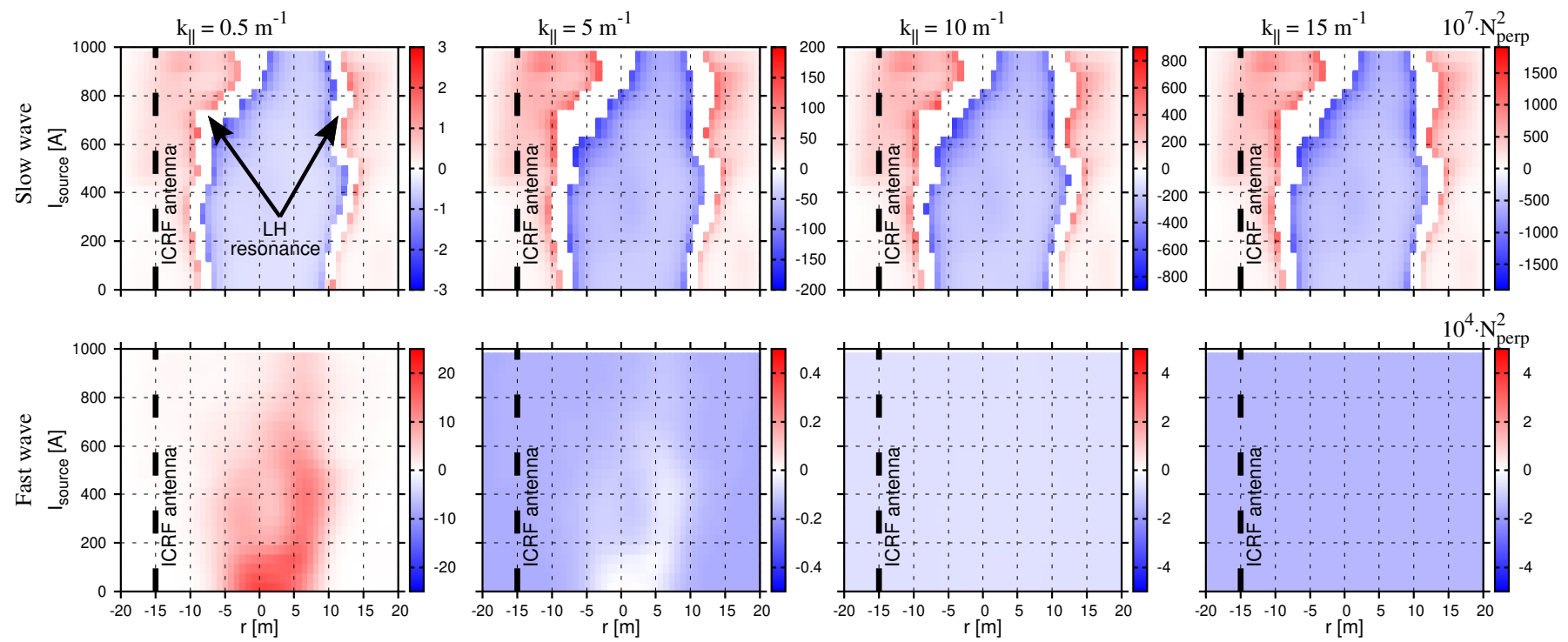

FIG. 12: The perpendicular component of the refraction coefficient in magnetised Ar plasma. Results are evaluated on the base of the plasma density radial profile in Fig. 9 corresponding to $I_{b i g}=2250 \mathrm{~A}$ and are represented in the same form. Results for slow (upper row) and fast (bottom row) waves are presented. Each column corresponds to a fixed value of $k_{\|}$, which is written on the top of each column. The wave frequency $\omega=6 \mathrm{MHz}$ corresponds to those routinely used in the IShTAR experiment. The sharp transition of $N_{\perp}^{2}$ for slow waves from red to blue region corresponds to the position of the lower-hybrid resonance.

quadratic equation with respect to the variable $N_{\perp}^{2}$, where $N_{\perp}$ is the perpendicular component of the refraction coefficient $N^{2}=\sqrt{N_{\perp}^{2}+N_{\|}^{2}}$. Therefore, this dispersion relation has in general two independent roots corresponding to two types of waves. In the frequency range $\omega<<\omega_{c e}\left(\omega_{c e}=2 \pi f_{c e}\right.$ is the electron cyclotron angular frequency) these waves are known as "Slow wave" and "Fast wave"28,29. The refraction coefficient for these waves depends on a number of parameters, such as $B, n_{e}$, gas type, wave frequency $\omega$, and the parallel component of the wave vector $k_{\|}=\frac{\omega}{c}$.

One of the first tasks of the IShTAR experiment is to study the phenomena in the ICRF antenna near field region. Therefore, chosen wave operating parameters should resemble the actual tokamak near-field behaviour. Figure 10 represents the distribution of $N_{\perp}^{2}$ for both slow and fast waves plotted as a function of electron density for parameters relevant to the edge region of tokamak plasmas: antenna frequency $\omega \approx 50 \mathrm{MHz}$, $B=2 T, k_{\|}=10 \mathrm{~m}^{-1}$ and for pure deuterium plasma. The choise of $k_{\|}=10 \mathrm{~m}^{-1}$ is based on the $k_{\|}$wavenumber spectrum of typical tokamak ICRF antenna evaluated in the near field region. For example, the two strap ASDEX Upgrade ICRF antenna has $k_{\|}$spectrum peaked at $k_{\|}=8.5 \mathrm{~m}^{-1}$. Under these conditions the lower hybrid resonance, which is considered to be the main cause of the high electric field in the near-field region, occurs around $n_{e} \approx 1 \times 10^{17} \mathrm{~m}^{-3}$ and is typically located in tokamak SOL. The fast wave does not propagate $\left(N_{\perp}^{2}<0\right)$ in a relatively low density range until $n_{e} \approx 1 \times 10^{18} \mathrm{~m}^{-3}$, when $N_{\perp}^{2}$ becomes positive. Practically fast waves are almost always evanescent in the SOL of a typical tokamak since plasma density typically achieved in the SOL never approaches cut-off value. Even though some plasma and operational parameters at IShTAR test facility differ from those in a tokamak SOL, the wave propagation for the typical IShTAR conditions is similar to that in a tokamak SOL. This can be verified comparing results presented in Fig. 10 and Fig. 11. The calculated distribution of $N_{\perp}^{2}$ for typical IShTAR conditions is shown in Fig. 11 as a function of plasma density for both slow and fast waves and for one particular $k_{\|}$value. Results are presented in a way similar to that in the figure 10. These results give a general impression of the wave behaviour in regions of different plasma density. Results correspond to following typical parameters: $\omega \approx 6 \mathrm{MHz}$, $B=0.1 T, k_{\|}=10 \mathrm{~m}^{-1}$. Again, the choice of $k_{\|}$is based on the $k_{\|}$wavenumber spectrum of the IShTAR ICRF antenna evaluated in the near field region. The IShTAR single strap antenna has a wide $k_{\|}$spectrum peaked at $k_{\|}=0$ and the spectral power reaches half of the peak value at $k_{\|}=10 \mathrm{~m}^{-1}$. That is why $k_{\|}=10 m^{-1}$ has been chosen as the most representative value. Meanwhile, this value is not so far from the typical $k_{\|}=8.5 \mathrm{~m}^{-1}$ of the ASDEX Upgrade ICRF antenna. The fast wave also does not propagate here in a relatively low density range until $n_{e} \approx 2 \times 10^{17} \mathrm{~m}^{-3}$. Looking ahead one must say that the typically achieved plasma density at IShTAR never exceed $n_{e} \approx 2.5 \times 10^{17} \mathrm{~m}^{-3}$ ( at least for the available at the moment RF power $3 \mathrm{~kW}$ ) implying that the fast wave is always evanescent for the given density range. This is similar to the wave propagation conditions in a tokamak SOL, as one can see from an analysis of the figure 10. The slow wave, in the other way, propagates at a low densities until the onset of the lower hybrid resonance at $n_{e} \approx 5 \times 10^{16} \mathrm{~m}^{-3}$, where $N_{\perp}^{2}$ undergoes a sharp transition from a very high positive to equally high negative values, after which the wave is evanescent. This is also similar to the typical situation in the tokamak SOL, as 
can be seen in Fig. 10 .

The corresponding resonance frequency is expressed as follows:

$$
\omega_{L H}^{2}=\frac{\omega_{c e} \omega_{c i}\left(\omega_{p e}^{2}+\omega_{c e} \omega_{c i}\right)}{\omega_{p e}^{2}+\omega_{c e}^{2}},
$$

where $\omega_{c e}$ and $\omega_{c i}$ are the electron and ion gyrofrequencies, respectively and $\omega_{p e}$ is the plasma frequency. For a fixed RF frequency $\omega$ the lower-hybrid resonance layer will be achieved at the density:

$$
n_{e}^{L H}=\frac{m_{e} \varepsilon_{0}}{e^{2}} \frac{\omega_{c e}^{2}\left(\omega_{c i}^{2}-\omega^{2}\right)}{\omega^{2}-\omega_{c e} \omega_{c i}},
$$

A thorough study of the operational domain for the wave propagation implies the interpretation of the results in Fig. 9 in terms of $N_{\perp}^{2}$ for different values of $k_{\|}$. The projection of $B$ and $n_{e}$ in the $N_{\perp}^{2}$ space allow us to understand and characterize the wave dynamics in the plasma edge and in the vicinity of the ICRF antenna strap. The aim is to find regions of propagation, evanescence, resonances and fast-to-slow wave conversion. These features will be more clear if one plots the more visual representation on the wave propagation in front of the ICRF antenna, namely the distribution of $N_{\perp}^{2}$ vs radial position. With this representation, the regions of propagating and evanescent waves are clearly visible.

The typical results are shown in Fig. 12. The analysis has been made for the wave frequency of $6 \mathrm{MHz} . N_{\perp}^{2}$ values are calculated on the base of density profiles shown in Fig. 9 corresponding to $I_{b i g}=2250 \mathrm{~A}$. The results are calculated for different values of $k_{\|}$(each column corresponds to different $k_{\|}$in the range of $0.5-15 \mathrm{~m}^{-1}$ ) since the natural $k_{\|}$spectrum of our one-strap ICRF antenna is relatively wide from 0 to approx. $30 \mathrm{~m}^{-1}$. The upper row corresponds to the slow wave while the bottom row corresponds to the fast one. The slow wave is propagating at the plasma edge up to the radial position $r \approx 10 \mathrm{~cm}$, where the sharp transition from a high positive to a high negative values is present. This transition corresponds to the onset of a lower-hybrid resonance.

Further inward from the position of the resonance the wave remains evanescent. For the slow wave the value $k_{\|}$plays a more quantitative role. For instance, no substantial difference in the position of the resonance layer for different $k_{\|}$is visible. Note, however, that the absolute value of $N_{\perp}^{2}$ strongly depends on $k_{\|}$, namely the higher $k_{\|}$the higher $N_{\perp}^{2}$. Ranges in first and last plots (corresponding to $k_{\|}=0.5 \mathrm{~m}^{-1}$ and $k_{\|}=15 \mathrm{~m}^{-1}$ ) have three orders of magnitude difference. Practically this leads to the situation that the propagation direction of slow wave gradually tilts towards the direction of $B$.

The fast wave remains evanescent in the entire plasma volume and almost for all values of $k_{\|}$except for a very narrow band of $k_{\|}$close to 0 , which fractionally contributes very little to the radiated RF power and is not of a practical interest. The results for other $I_{b i g}$ do not show a significantly different picture. Only the position of the lower-hybrid resonance shifts slightly either inwards or outwards depending on the details of the plasma density profile. These results essentially resemble the tokamak near field region, where fast wave is evanescent and only slow wave can transfer the power up to the position of the lower-hybrid resonance where it accelerates particles causing hot spots, arcing and sputtering.

In order to study the role of the lower-hybrid resonance on the phenomena close to the antenna launcher one needs to shift the position of the resonance to the direct vicinity of the ICRF antenna strap. This can be done accordingly by tailoring the plasma density radial profile and magnetic field while changing the magnetic field distribution $I_{b i g}$ and $I_{\text {source }}\left(B\right.$ and $n_{e}$ are coupled through the magnetic mapping and other effects). Results in Fig. 13 give a direct recipe on how to choose the proper window in parameter space in order to shift the slow wave resonance layer to the necessary position near the antenna. The perpendicular component of the refraction coefficient in a magnetised $A r$ plasma is presented for $k_{\|}=10 \mathrm{~m}^{-1}$. Results are evaluated on the base of the plasma density radial profile in Fig. 9 such that each column corresponds to a fixed value of $I_{b i g}$, which is written on top of each column. The wave frequency $\omega=6 \mathrm{MHz}$ corresponds to those routinely used in the IShTAR experiment. These results suggest that the optimum condition would be $I_{b i g}>3000 \mathrm{~A}$ and $I_{\text {source }}>500 \mathrm{~A}$, where the position of resonance directly approaches the antenna launcher. The corresponding results for fast wave are not presented since fast wave always evanescent $\left(N_{\perp}^{2} \sim-4 \times 10^{4}\right)$ within the parameter domain considered in this figure.

\section{SIMULATIONS AND MEASUREMENTS OF THE ICRF ANTENNA FIELD}

Numerical simulations are performed for IShTAR in COMSOL software. The focus of the simulations is on the ICRF antenna fields. The COMSOL IShTAR model includes all main features of the device and a precise geometrical representation of the ICRF antenna. In Fig. 14 the magnetic field distribution in vacuum is shown on a cut plane. It is seen that the field is the strongest in the coaxial line, at its exit and in the region between the antenna strap and the back wall behind it. It was also observed during experiments that strong visible light radiation is present at the same locations. Experimental measurements of ICRF magnetic field components were done using B-dot probes and these probes were also replicated in the model. Radial profiles of vacuum magnetic fields from simulations and from experiments were compared and showed good agreement, which validates the geometry of IShTAR reproduced in the modelling ${ }^{30}$. The next step is the transition from vacuum to plasma simulations. The performed simulations of wave propagation in plasma allow us to see the 3D wave structure that is expected in IShTAR. The propagating slow wave influences directly the values of the electric field in the RF sheath. The work on simulations with plasma has been done for the conditions typical for IShTAR in a simplified geometry ${ }^{31}$. Obtained qualitative dependency of the global RF electric field structure on the plasma conditions 


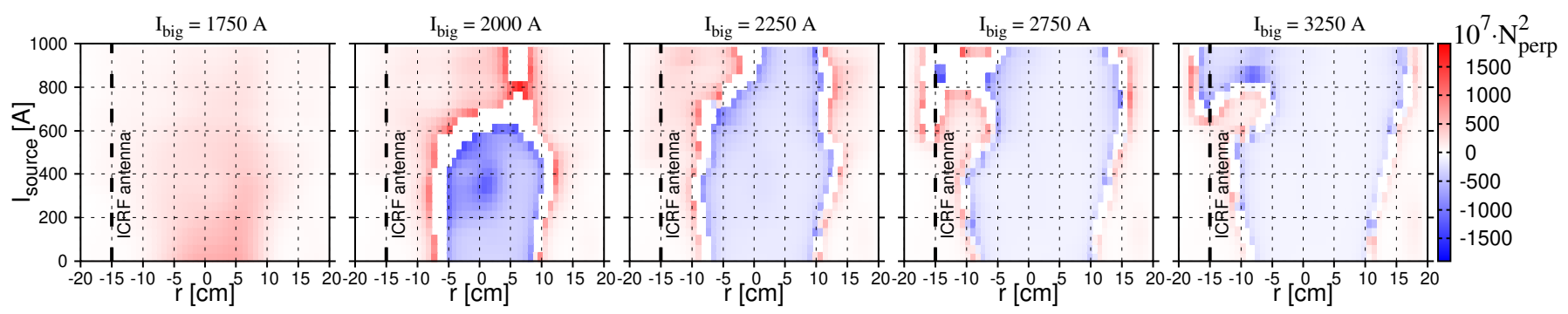

FIG. 13: The perpendicular component of the refraction coefficient in magnetised $A r$ plasma for $k_{\|}=10 \mathrm{~m}^{-1}$. Results for slow wave are presented. Results are evaluated on the base of the plasma density radial profile in Fig. 9. Each column corresponds to

a fixed value of $I_{b i g}$, which is written on the top of each column. The wave frequency $\omega=6 \mathrm{MHz}$ corresponds to those routinely used in the IShTAR experiment. The sharp transition of $N_{\perp}^{2}$ for slow waves from red to blue region corresponds to the position of the lower-hybrid resonance. These results suggest that the optimum condition to shift the resonance layer in the direct vicinity of the ICRF antenna launcher would be $I_{\text {big }}>3000 \mathrm{~A}$ and $I_{\text {source }}>500 \mathrm{~A}$.

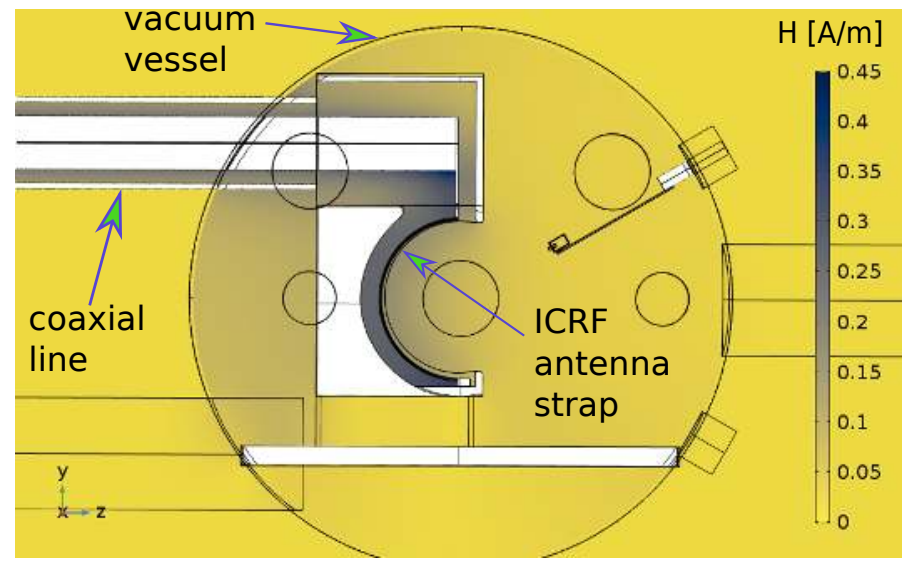

FIG. 14: The COMSOL model of the magnetic field distribution in IShTAR in the cut plane intersecting the ICRF antenna.

provides the idea of the best scenarios for RF sheath measurements on the antenna limiters. Output of the parallel electric field in front of the ICRF antenna could be used as an input for SSWICH (Self-consistent Sheaths and Waves for Ion Cyclotron Heating) code $^{32}$ to calculate the distribution of the RF sheath rectified voltages in the assumptions of the code.

The optical spectroscopy is used as a main diagnostic tool for measuring the electric fields in the vicinity of ICRF antenna. Recent progress on the passive optical emission spectroscopy measurements gave the electric field strength in the order of $3.4 \pm 0.3 \mathrm{kV} / \mathrm{cm}$ for $1 \mathrm{~kW}$ of applied RF power. More details about these measurements are presented in Ref. ${ }^{23}$.

\section{CONCLUSION}

A new ICRF test facility has been developed to study phenomena near an ICRF antenna launcher. It has a number of dedicated diagnostic ports making region near an ICRF antenna thoroughly accessible for numerous diagnostics. Several features have been discussed related to Langmuir probe and magnetic measurements in RF plasmas. In particular, a simplified version of an "RF compensated probe", which does not require a secondary electrode and frequency selective magnetic measurements scheme. The potential of the experiment to reproduce the same wave propagation scenario near an ICRF antenna launcher as in a large fusion experiment has been demonstrated. The presented setup has a potential application much broader than simply ICRF relevant studies. A large diameter of the main vessel $(\sim 1 \mathrm{~m})$, range of magnetic field (up to $0.3 \mathrm{~T}$ ) and achievable plasma densities (up to $5 \times 10^{17} \mathrm{~m}^{-3}$ ) makes it suitable for diverse fields in fundamental and applied plasma research. The team of IShTAR looks forward to and encourages new fruitful collaborations.

\section{ACKNOWLEDGMENTS}

This work has been carried out within the framework of the EUROfusion Consortium and has received funding from the Euratom research and training programme 2014-2018 and 2019-2020 under grant agreement No 633053. The views and opinions expressed herein do not necessarily reflect those of the European Commission.

${ }^{1}$ S. Devaux, E. Faudot, J. Moritz, and H. S., Nuclear Materials and Energy 12, 908-912 (2017).

${ }^{2}$ E. Faudot, S. Devaux, J. Moritz, S. Heuraux, P. Molina Cabrera, and F. Brochard, Review of Scientific Instruments 86, 063502 (2015).

${ }^{3}$ K. Crombé et al., (2016), 26th IAEA Fusion Energy Conference.

${ }^{4}$ D. G. Miljak and F. F. Chen, Plasma Sources Sci. Technol. 7, 61 (1998).

${ }^{5}$ M. Light and F. F. Chen, Physics of Plasmas 2, 1084 (1995).

${ }^{6}$ F. F. Chen, Plasma Sources Sci. Technol. 24, 014001 (2015).

${ }^{7}$ F. F. Chen, Plasma Phys. Control. Fusion 33, 339 (1991).

${ }^{8}$ I. D. Sudit and F. F. Chen, Plasma Sources Science and Technology 3, 162 (1994).

${ }^{9}$ C. Bowick, RF Circuit design (Newnes, Burlington, 1982).

${ }^{10}$ T. Lafleur, C. Charles, and R. W. Boswell, J. Phys. D: Appl. Phys. 44, 055202 (2011).

${ }^{11}$ T. A. Lafleur, Helicon Wave Propagation in Low Diverging Magnetic Fields, Ph.D. thesis, The Australian National University (2011).

${ }^{12}$ K. K. Barada, P. K. Chattopadhyay, J. Ghosh, S. Kumar, and Y. C. Saxena, Physics of Plasmas 20, 042119 (2013).

${ }^{13}$ A. W. Degeling, Physics of Plasmas 10, 2586 (2003).

${ }^{14}$ A. W. Degeling, C. O. Jung, R. W. Boswell, and A. R. Ellingboe, Physics of Plasmas 3, 2788 (1996). 
IShTAR: a test facility to study the interaction between RF wave and edge plasmas

${ }^{15}$ G. Sato, W. Oohara, and R. Hatakeyama, Plasma Sources Sci. Technol. 16, 734-741 (2007).

${ }^{16}$ C. M. Franck, O. Grulke, and T. Klinger, Physics of Plasmas 10, 323 (2003).

${ }^{17}$ I. Sudit and F. Chen, Plasma Sources Sci. Technol. 3, 162 (1994).

${ }^{18}$ A. Ganguli, B. Sahu, and T. R.D., Plasma Sources Sci. Technol. 17, 015003 (2008)

${ }^{19}$ I. Shesterikov, A. V. Stechow, O. Grulke, R. Stenzel, and T. Klinger, Journal of Instrumentation 12, T07009 (2017)

${ }^{20}$ U. Tietze, C. Schenk, and E. Gamm, Electronic Circuits. Handbook for Design and Application (Springer, Heidelberg, 1991).

${ }^{21} \mathrm{P}$. Horowitz and W. Hill, The Art of Electronics. Third edition (Cambridge University Press, New York, 2015).

${ }^{22}$ J. B. Hagen, Radio-Frequency Electronics: Circuits and Applications (Cambridge University Press, New York, 1996).

${ }^{23}$ A. Kostic et al., Review of Scientific Instruments 89, 10D115 (2018).

${ }^{24} \mathrm{~T}$. Verstrynge, Spectroscopic diagnostics of IShTAR plasma, Master's thesis, Ghent University, Faculty of Science (2018).
${ }^{25}$ S. N. Bathgate, M. Bilek, and D. R. Mckenzie, Plasma Science and Technology 19, 083001 (2017).

${ }^{26}$ E. O. Åström, Arkiv. Fysik 2, 443 (1950).

${ }^{27}$ W. P. Allis, Waves in Anisotropic Plasmas (MIT Press, Cambridge, 1963).

${ }^{28}$ T. H. Stix, Waves in Plasmas (Springer Science \& Business Media, New York, 1992).

${ }^{29}$ K. Crombé and D. Van Eester, Journal of Plasma Physics 82, 905820203 (2016).

${ }^{30}$ M. Usoltceva, R. Ochoukov, R. D’Inca, J. Jacquot, K. Crombé, A. Kostic, S. Heuraux, E. Faudot, and J.-M. Noterdaeme, EPJ Web of Conferences 157, 03058 (2017).

${ }^{31}$ M. Usoltceva, Ph.D. thesis, Ghent University (In preparation).

${ }^{32} \mathrm{~J}$. Jacquot, Non-linear self-consistent description of radiofrequency wave propagation and the periphery of a magnetized plasma, $\mathrm{Ph} . \mathrm{D}$. thesis, The University of Lorraine (2013). 